\title{
Superfluidity of Bose-Einstein condensates in toroidal traps with nonlinear lattices
}

\author{
A. V. Yulin ${ }^{1}$, Yu. V. Bludov ${ }^{2}$, V. V. Konotop ${ }^{1}$, V. Kuzmiak ${ }^{3}$, M. Salerno ${ }^{4}$ \\ ${ }^{1}$ Centro de Física Teórica e Computacional and Departamento de Física, Faculdade de Ciências, \\ Universidade de Lisboa, Avenida Professor Gama Pinto 2, Lisboa 1649-003, Portugal \\ 2 Centro de Física, Universidade do Minho, Campus de Gualtar, Braga 4710-057, Portugal \\ 3 Institute for Photonics and Electronic of Czech Academy of Science, Chaberska 57, 182 51, Praha 8, Czech Republic \\ ${ }^{4}$ Dipartimento di Fisica "E.R. Caianiello", Università di Salerno, \\ via ponte don Melillo, 84084 Fisciano (SA), Italy
}

(Dated: September 1, 2018)

\begin{abstract}
Superfluid properties of Bose-Einstein condensates (BEC) in toroidal quasi-one-dimensional traps are investigated in the presence of periodic scattering length modulations along the ring. The existence of several types of stable periodic waves, ranging from almost uniform to very fragmented chains of weakly interacting and equally spaced solitons, is demonstrated. We show that these waves may support persistent atomic currents and sound waves with spectra of Bogoliubov type. Fragmented condensates can be viewed as arrays of Josephson junctions and the current as a BEC manifestation of the dc-Josephson effect. The influence of linear defects on BEC superfluidity has been also investigated. We found that for subcritical velocities, linear defects that are static with respect to the lattice (while the condensate moves in respect to both the optical lattice and the defect) preserve the BEC superfluidity.
\end{abstract}

PACS numbers: 00.00.AA

\section{INTRODUCTION}

The possibility of particles flows in multiply connected superfluid and superconducting systems which can last for extraordinary long, in principle infinitely long, times is certainly one of the most striking manifestation of quantum mechanics at the macroscopic level [1]. This fact, well known for conventional superconductors and superfluids, has been recently observed also in atomic Bose-Einstein condensates (BEC) confined in toroidal traps [2, 3], with persisting circulating currents. In addition to the fundamental interest of this result as a direct manifestation of the BEC superfluidity [4], matter waves in multiply connected geometries also represent ideal systems for exploring nonlinear properties in the presence of nonzero density backgrounds. In this context, solitary matter waves in one-dimensional (1D) and $2 \mathrm{D}$ toroidal traps have been investigated and the existence of a bandgap structure and of gap solitons have been reported in Ref. [5]. Possibility of management of solitons, including acceleration and localization of matter waves, was explored in Ref. [6]. The density distributions in a ring as a function of the relation between the healing length and the trap length was investigated in Ref. [7]. Solitary waves have also been studied for quasi $1 \mathrm{D}$ circular troughs of radially periodic $2 \mathrm{D}$ potentials 8].

All these studies refer to the case of uniform or periodic linear potentials present along the circumference of the trap. Moreover, several experimental studies of BEC in linear optical lattices (OL) have shown the occurrence of interesting phenomena related to phase relationships between BEC wavefunctions in different sites, these including the existence of Josephson currents [9], the proliferation of vortices in the Berezinskii-Kosterlitz-Thouless regime of BECs in 2D OLs [10], vortex nucleation in ro- tating lattices of BECs [11], and so on.

On the other hand, it is known that interesting phenomena also arise when a linear optical lattice is combined with a periodic spatial modulation of the nonlinearity, also known as a nonlinear OL. This is particularly true for 1D settings for which it has been shown that periodic spatial modulations of the scattering length, achieved with the help of the Feshbach resonance technique [12], can induce long lived Bloch oscillations 13], Rabi oscillations [14], and dynamical localization [15] of gap solitons in the presence of accelerated linear periodic potentials. Periodic modulations of the nonlinearity have also been shown to be effective to generate solitons in $1 \mathrm{D}$ OLs [16] and in random potentials [17] to stabilize multidimensional solitons [18] and to generate vortex rings in a highly controllable manner [19] (for fresh review of this very active field of investigation see Ref. [20]).

We remark that toroidal traps are routinely created in laboratories with the aid of magnetic fields [21] and nonlinear OLs have also been recently experimentally realized for BEC in linear settings [22]. Methods to create trapping potentials for BECs by means of rapidly moving laser beams for a variety of geometries, including toroids, ring lattices and square lattices, have also been recently developed [23].

To our knowledge, nonlinear OLs with a multiply connected geometry have not yet been realized. Considering the experimental rapid progresses in the field, however, it is reasonable to expect them to be soon available. A nonlinear toroidal lattice could indeed be created by optically induced Feshbach resonances [24] using an all-optical trap with an horizontal sheet beam and a ring shaped vertical higher order Gauss-Laguerre beam [25] whose intensity is periodically modulated along the ring due to phase interference (see, e.g., Ref. [26]). This setting would be especially effective in a $1 \mathrm{D}$ limit (for details of respective 
reduction see, e.g., Ref. [6]) and would permit to study the combined effects of nonlinearity and periodicity on the BEC superfluidity, a problem scarcely investigated in the literature.

The aim of this paper is to study superfluid properties of BECs in toroidal traps with strong radial confinement and periodic modulations of the scattering length (nonlinearity) in the azimuthal direction in the framework of the mean field Gross-Pitaevskii equation (GPE). In particular, we demonstrate the existence of several types of stable periodic waves, ranging from almost uniform to very fragmented chains of weakly interacting and equally spaced solitons, which can support persistent currents and permit sound waves with interesting physical properties. The backgrounds that may exist in such traps can be classified in terms of bifurcation diagrams in the parameter space $(\mu, \rho)$, where $\mu$ is the chemical potential and $\rho$ is the density of the condensate. We analyze the bifurcations patterns and the stability properties of periodic matter waves both with zero (stationary states) and nonzero particle currents. The spectral properties of the linear excitations (sound waves) propagating in periodic condensates are also investigated and the Bogoliubov type features of the spectrum demonstrated. In the presence of a pronounced fragmentation of the condensate (i.e., in the soliton chain limit) we find that persistent currents of particles are expressed in the form $\rho_{\min } \sin \theta$, where $\rho_{\text {min }}$ is the minimum density of the condensate and $\theta$ is the phase difference of the order parameter of neighboring solitons. Quite interestingly, this expression is similar to the supercurrent expression of a Josephson junction in the zero voltage state (dc- Josephson effect), this suggesting the interpretation of fragmented condensates as arrays of Josephson junctions and the persistent current as a BEC analog of the dc-Josephson effect 27]. Superfluidity properties are also investigated in the presence of localized linear defects perturbing the nonlinear OL and acting on the condensate as obstacle. Direct numerical simulations show that, in conformity with the Landau criterion and in agreement with our sound wave analysis, the BEC superfluidity is preserved in presence of linear defects which are static with respect to the nonlinear OL and that move in respect to the condensate with subcritical velocities.

The paper is organized as follows. In Sec. [II we formulate the mathematical model describing the condensate and analyze the ground states properties. Families of periodic solitons in parameter space and their stability properties both for zero (stationary) and non zero carrying current states are discussed in in Secs. II A and II $\mathrm{B}$, respectively. The spectra of sound waves in the condensate and its Bogoliubov structure are numerically calculated and discussed in Sec. III. In Sec. IV the superfluid stability properties of BEC are investigated by direct numerical integrations of the GPE in the presence of a linear defect. Finally, in the Sec.V, the main results of the paper are summarized.

\section{MODEL EQUATION}

We consider the following dimensionless GP equation

$$
i \psi_{t}=-\psi_{x x}+U(x)|\psi|^{2} \psi+V(x) \psi,
$$

where $U(x)$ is a periodical nonlinear potential that accounts for interatomic interactions and $V(x)$ is a localized linear potential modeling an obstacle perturbing the flow of the ground state. Without loss of generality, the period of the nonlinear lattice is chosen to be $\pi$, i.e. $U(x+\pi)=U(x)$ and the circumference of the trap is fixed to $L=M \pi$, with $M$ being a positive integer. The annular geometry obviously implies cyclic boundary conditions

$$
\psi(x, t)=\psi(x+L, t),
$$

and permits the existence of current carrying states, with the current, $J$, defined as

$$
J=\frac{1}{2 i}\left(\psi^{*} \psi_{x}-\psi \psi_{x}^{*}\right)
$$

(here the asterisk denotes complex conjugation). The properties of these states are investigated in the following two subsections.

\section{A. Ground states with zero current}

We start from the the analysis of the stationary states having zero current: $J=0$, assuming that no linear defect is added to the system: $V(x) \equiv 0$. In this case the order parameter has a constant phase, and can be searched in the form $\psi=\Psi(x) e^{-i \mu t}$, where $\mu$ is the chemical potential and $\Psi$ is real and solves the stationary GP equation

$$
\mu \Psi=-\Psi_{x x}+U(x) \Psi^{3} .
$$

We are particularly interested in nonlinear potentials changing the sign of the interaction, i.e. in the situations in which the repulsive and attractive interactions alternate along the length $L$ of the trap. We remark that for a priori fixed particular solutions $\Psi(x)$ it is possible to construct exact potentials $U(x)$ for Eq. (4) using the "inverse engineering" technique explained in Ref. 28]. An example of such a potential is given by

$$
U(x)=\frac{\kappa q^{2}}{4} \frac{\operatorname{sn}(q x, \kappa)(3 \kappa \operatorname{sn}(q x, \kappa)-2)}{1+\kappa \operatorname{sn}(q x, \kappa)}, \quad q=\frac{4 K(\kappa)}{\pi}
$$

with sn denoting the standard Jacobi elliptic function and $K(\kappa)$ the elliptic integral. Notice that $U(x)$ is parametrized by the elliptic modulus $\kappa \in[0,1]$ and depending on the value of $\kappa$, it represents either a sequence of nonlinear single wells (for $0<\kappa<\sqrt{5 / 3}-1$ ) or a series of nonlinear double wells (for $\sqrt{5 / 3}-1<\kappa<1$ ). In this paper we shall restrict our study to the case 
of single-well potentials, only. We also remark that in spite of the apparent complexity of the potential (5), it can be approximated very well by a Fourier series with only few harmonics. Indeed, even for the limiting value $\kappa=\sqrt{5 / 3}-1$ we obtain for the coefficients of the Fourier series $U(x)=\langle U\rangle+\sum_{k \neq 0} c_{k} e^{2 i k x}$, where $\langle U\rangle \approx 0.239$, $\left|c_{ \pm 1}\right| \approx 0.36,\left|c_{ \pm 2}\right| \approx 0.12,\left|c_{ \pm 3}\right| \approx 0.016,\left|c_{ \pm 4}\right| \approx 0.001$, and so on. This implies that the suggested periodic modulation of the nonlinearity can be simulated with accuracy of about $5 \%$ by employing only two laser beams with the wavelengths $\pi$ and $\pi / 2$ in dimensionless units.

An exact analytic solution of Eq. (4) with $V=0$ and with the potential $U(x)$ taken as in Eq.(5), can be written as

$$
\Psi_{a}=\sqrt{1+\kappa \operatorname{sn}(q x, \kappa)},
$$

with the corresponding chemical potential given by $\mu_{a}=$ $\kappa^{2} q^{2} / 4$. This solution is just a member of a family of periodic solutions which are parameterized by the chemical potential $\mu$ and identified by the period $L_{0}$. Note that although $L_{0}$ does not need to coincide with the period of the nonlinear potential $U(x)$, a relation $L=m L_{0}$ with $m$ integer, must be obviously satisfied. Since we have fixed $L=M \pi$ the set of possible periods of the solutions is given by all $m$, which are integer divisors of $M$, i.e., $M / m$ is an integer and, respectively, $L_{0}=(M / m) \pi=p \pi$. In Fig. 1 we illustrate the bifurcation diagrams of these family solutions obtained numerically in the parameter space $(\mu, \rho)$, where $\rho=\left\langle|\Psi|^{2}\right\rangle$ is the linear density of the condensate 31] denoting the average of any $L_{0}$-periodic function $f(x)$ as $\langle f\rangle=\frac{1}{L_{0}} \int_{0}^{L_{0}} f(x) d x$, we have found that the potential (5) satisfies $\langle U\rangle>0$, i.e. the potential is repulsive in average. In the present paper we restrict mainly to branches bifurcating from linear solutions for which the limiting transition $\rho \rightarrow 0$ is possible. In this limit the ground state bifurcates from the uniform solution $\Psi_{c}=\sqrt{\mu} / \sqrt{\langle U\rangle}+\mathcal{O}\left(\mu^{3 / 2}\right)$ and the respective branch has the period $\pi$. The bifurcation points of the other solutions having linear limit can be designated by $\mu_{m}=4 m^{2} / M^{2}$. In each of such points, however there are two emergent modes: cos- and sin-like modes:

$$
\begin{gathered}
\Psi_{c} \approx \sqrt{\frac{\mu-\mu_{m}}{\frac{2}{\pi} \int_{0}^{\pi} U\left(\frac{M x}{m}\right) \cos ^{4}(2 x) d x}} \cos \left(\frac{2 m}{M} x\right), \\
\Psi_{s} \approx \sqrt{\frac{\mu-\mu_{m}}{\frac{2}{\pi} \int_{0}^{\pi} U\left(\frac{M x}{m}\right) \sin ^{4}(2 x) d x}} \sin \left(\frac{2 m}{M} x\right) .
\end{gathered}
$$

Note that, formally, the ground state can be obtained from Eq.(77) by putting $m=0$.

From Figs. 1(a) and 1(b) we observe that an increase of the ground state density leads to the growth of the chemical potential, as is expected for a BEC with repulsive interatomic interactions. However, when the number of particles increases the nonlinear interactions become important and the condensate becomes denser in the areas of attractive interactions. At the point where the (a)

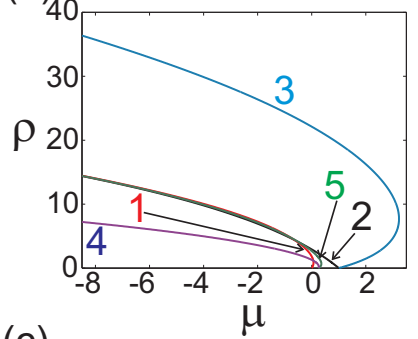

(c)

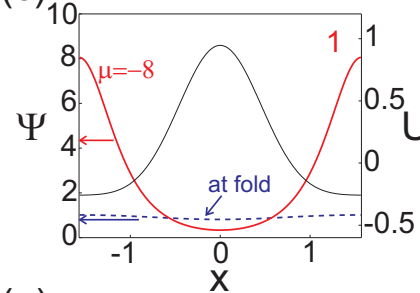

(e)

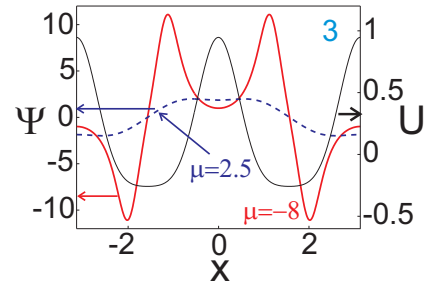

(d)

(b)
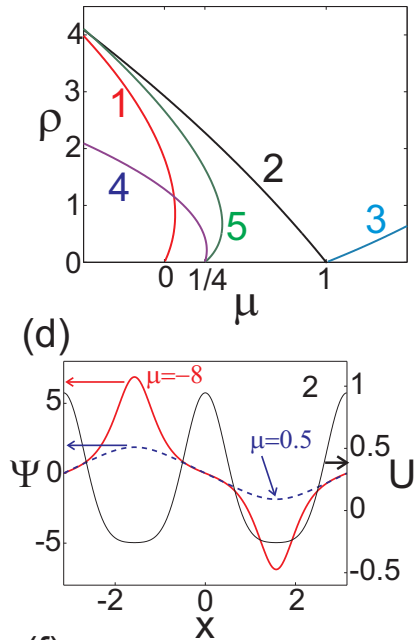

(f)

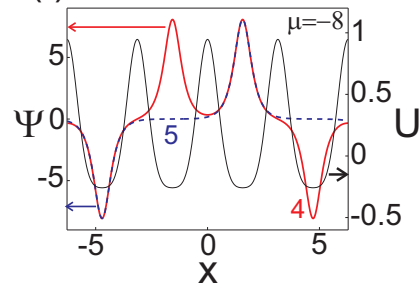

FIG. 1: (Color online) [(a) and (b)] Families of static solutions for the potential (5) centered at $x=\pi / 2$ with $\kappa=0.25$ [solid black lines in panels (c)-(f)] bifurcating from linear modes for the cases:

$L_{0}=\pi$ [red line (1)], $L_{0}=2 \pi$ [black (2) and cyan (3) lines], and $L_{0}=4 \pi$ [purple (4) and green (5) lines]. (c) The order parameter of the solutions is shown in panels (a) and (b) by red line (1). The solid red curve corresponds to the solution with $\mu=-8$ and the solution at the folding point $\mu=0.08$ is shown by the dashed blue line. (d) The order parameter of the solutions is shown in (a) and (b) by the black line (2)

for $\mu=-8$ (solid red line) and for $\mu=0.5$ (dashed blue line). (e) The same as in panel (c) but for the solutions corresponding to $\mu=2.5$ (dashed blue line) and $\mu=-8$ (solid red line) of the family shown by cyan line (3). (f) The same as in panel (c) but for the solution corresponding to $\mu=-8$ (dashed blue line) of the family shown by the purple line (4) and for the solution corresponding to $\mu=-8$ (solid red line) of the family shown by the green line (5).

bifurcation diagram turns back, referred below as folding point, the chemical potential decreases [see the red line (1) in Figs. 1(a) and 1(b)], and, for some average density, the chemical potential becomes negative. This happens because for larger densities most of the particles concentrate in the areas with attractive interactions and, therefore, the whole condensate behaves as having a negative scattering length. Mathematically, this is clear from the relation

$$
\mu \rho=H=\left\langle\Psi_{x}^{2}\right\rangle+\left\langle U \Psi^{4}\right\rangle
$$

from which we see that a negative $\mu$ implies $\left\langle U \Psi^{4}\right\rangle<0$ 
(a)

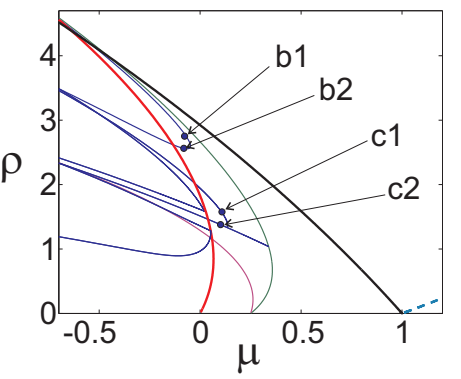

(b)

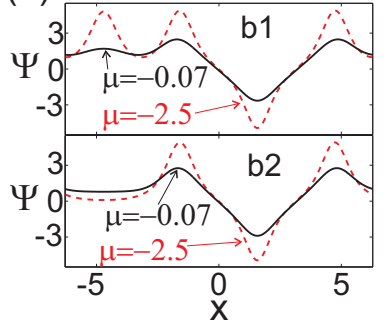

(c)

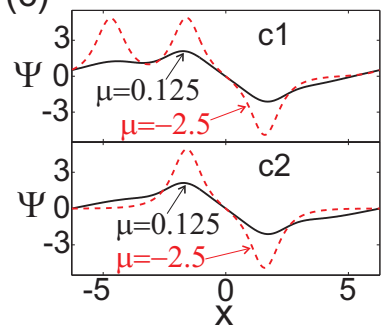

FIG. 2: (a) Solid lines show the dependencies of the linear density on the chemical potential for the solutions with $L_{0} \leq 4 \pi$ and having no more than one bright soliton in each area of attractive interaction. The thick black and red lines correspond to the modes bifurcating from the linear solutions with the periods $\pi$ and $2 \pi$ and, at large negative $\mu$, having one soliton in each area of the attractive interactions. The other two modes originating from linear ones are shown by the green and purple lines. Thin blue lines show the dependencies for the solutions that do not have a linear limit. The dashed thick cyan line shows a bifurcation curve which corresponds to the solutions originating from a linear one and having two solitons in each area of the attractive interactions. (b) The field distributions for the solution corresponding to the points of the bifurcation curve denoted by b1 and b2 in panel(a) $(\mu=-0.07)$ (black solid line). The red dashed lines show the solution with $\mu=-2.5$ and belonging to the same bifurcation branches. (c) The same as in (b) but for the points marked in panel (a) by the circles $\mathrm{c} 1$ and $\mathrm{c} 2$; the chemical potentials in this case are $\mu=0.125$.

(note, that in the folding point $\mu_{*}$, where $d \mu / d \rho=0$ we have the relations $\left.\mu_{*}=\frac{H}{\rho}=\frac{d H}{d \rho}\right)$. Thus a condensate with sufficiently large density can be considered as an array of in-phase bright matter solitons located at the areas with negative scattering length; see Fig. 1(c). Obviously, the interaction between the solitons decays exponentially as the density of the condensate increases, transforming in an array of in-phase matter solitons.

On the other hand, from the diagram bifurcating from the point $\mu=1$ [black (2) and blue (3) lines in Figs. 1(a) and 1(b)] we observe that the solution bifurcating from the sin mode [Eq.(77)] at large negative $\mu$ can be seen as a chain of out-of-phase $(\ldots 0-\pi-0-\pi \ldots)$ bright solitons. The solutions belonging to the second branch bifurcating from Eq.(8) in the strongly nonlinear limit also transform into a chain of pairs of bright solitons; in this case there are two matter solitons in each domain of attractive interactions.
It is worth pointing out that the solution families shown in Fig. 11 reveal a peculiar feature, namely the kinetic energy of the condensate at the folding point is much larger than the potential energy $\left\langle\Psi_{x}^{2}\right\rangle \gg\left\langle U \Psi^{4}\right\rangle$. We also note that not all periodical solutions bifurcate from linear modes: There are branches having no zero nonlinearity limit which is however, expectable in view of the analogous behavior of the localized modes [29]. Their bifurcation diagrams have folding points or they merge with other solutions. In Fig. 2 the bifurcation diagrams are shown for all possible solutions which in the strongly nonlinear limit have no more than one soliton per period of the nonlinear potential and the period of these solutions is smaller than four periods of the nonlinear potential.

\section{B. Periodic matter waves with non zero current}

We now consider periodic states carrying nonzero currents $J \neq 0$. Such states can be represented in the form

$$
\Psi(x)=\sqrt{\rho(x)} e^{i \theta(x)}, \quad \theta(x)=\int v(x) d x,
$$

where $v(x)$ is the superfluid velocity and $\rho(x)$ the condensate density. It is straightforward to show that such solutions exist only if $J=v(x) \rho(x)=$ const [see the definition, Eq.(3)], i.e. if $d J / d x=0$, with the density $\rho$ satisfying the equation

$$
\frac{1}{2} \frac{d^{2} \rho}{d x^{2}}-\frac{1}{4 n}\left(\frac{d \rho}{d x}\right)^{2}+\mu \rho-\frac{J^{2}}{\rho}-U(x) \rho^{2}=0
$$

(note that zero-current states are characterized by densities which are strictly positive so the above equation is nonsingular). In the following we consider $L_{0}$-periodic density distributions

$$
\rho\left(x+L_{0}\right)=\rho(x)
$$

of the form $\rho(x)=\rho+\rho_{1}(x)$, with $\rho=\langle\rho(x)\rangle$ the mean density and $\rho_{1}(x)$ a periodic variation of the density of period $L_{0}$. The condition $J=$ const then implies that also the hydrodynamic velocity is of the form $v(x)=v_{0}+$ $v_{1}(x)$ with $v_{0}=\langle v\rangle$ the average superfluid velocity and $v_{1}$ a periodic function of period $L_{0}$. The order parameter $\Psi(x)$ has the form of a nonlinear Bloch state

$$
\Psi_{v_{0}}(x)=e^{i v_{0} x} f_{v_{0}}(x)
$$

with the mean velocity $v_{0}$ playing the role of quasimomentum and with $f_{v_{0}}(x)$ being a complex periodic function of period $L_{0}$. For the single valuedness or the wavefunction $\Psi(x)$, the average superfluid velocity must be quantized with respect to the length $L$ of the ring according to

$$
v_{0}=\frac{2 \pi p}{L}=\frac{2 p}{M}
$$



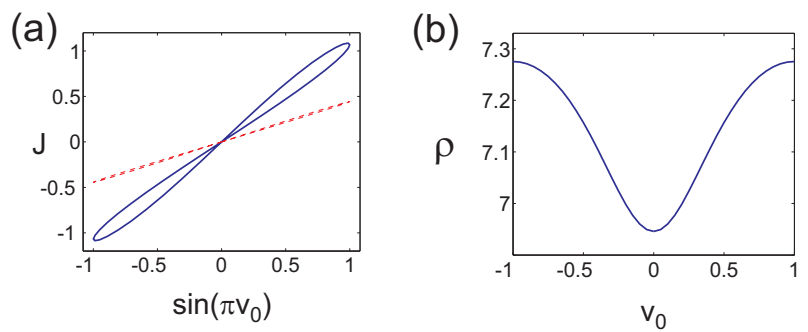

FIG. 3: (a) The current $v$ s the average velocity for $\mu=-2$ (blue curve) and $\mu=-8$ (red curve). (b) The average density $v s$ the average velocity for $\mu=-2$.

where $p$ is an integer. We should remark that the analogy exploited above between superfluids in a ring and Bloch electrons in crystals was first discussed by Bloch [30] and further developed in Ref. [5] for BEC in rotating traps. Obviously, in the linear limit $f_{v_{0}}(x)$ is simply reduced to $\sqrt{\rho}$, and, taking into account, that in this limit $\rho \rightarrow 0$, one can neglect the spatial variations of the density and conclude that the bifurcation curves start at $\mu=v_{0}^{2}$.

It is of interest to investigate how the condensate with a nonzero current behaves when its average velocity $v_{0}$ is changed. This is done in Fig. 3 where the current and the average density versus the average velocity are depicted for two fixed values of the chemical potential. Note that the current depends on the average superfluid velocity periodically, acquiring maxima at semi-integer values $v_{0}=p / 2$, while at integer values of $v_{0}$ the current vanishes. This is a direct consequence of the Bloch form (13) of the wavefunction implying that nondegenerate states with given $\mu$ and $v_{0}$ are equivalent to the state with the same chemical potential $\mu$ but with the velocity $v_{0}+p$, where $p$ is an integer. Also note that at $v_{0}=0$ the solution in Fig. 3 is carrying zero current [as the symmetric state belonging to the bifurcation branch (1)] while at $v_{0}=1$ the obtained periodic state is the asymmetric mode belonging to the branch (2) shown in Fig. 1 which is also a zero-current state.

Another interesting property to remark is the quasi sinusoidal dependence of the current $J$ on $v_{0}$ for BEC wavefunctions fragmented as chains of solitons on a nonzero background. The particle density of such condensates is depleted (enhanced) in correspondence with the regions of repulsive (attractive) interaction. Direct numerical simulations show that the stronger is the depletion of the condensate in the repulsive regions the better the current is approximated by a sinusoidal law. In the limit of very strong depletion (i.e., for a chain of strongly localized and weakly overlapping solitons), it becomes

$$
J \approx \frac{1}{2} \sqrt{-\mu} \rho_{\min } \sin \theta
$$

where $\rho_{\min }=-8 \mu / \max (U) \cdot \operatorname{sech}^{2}(\sqrt{-\mu} \pi / 2)$ denotes the minimum density of the condensate and $\theta$ is the phase difference of the order parameter between neighboring solitons. We also note that in the case of strong localiza-

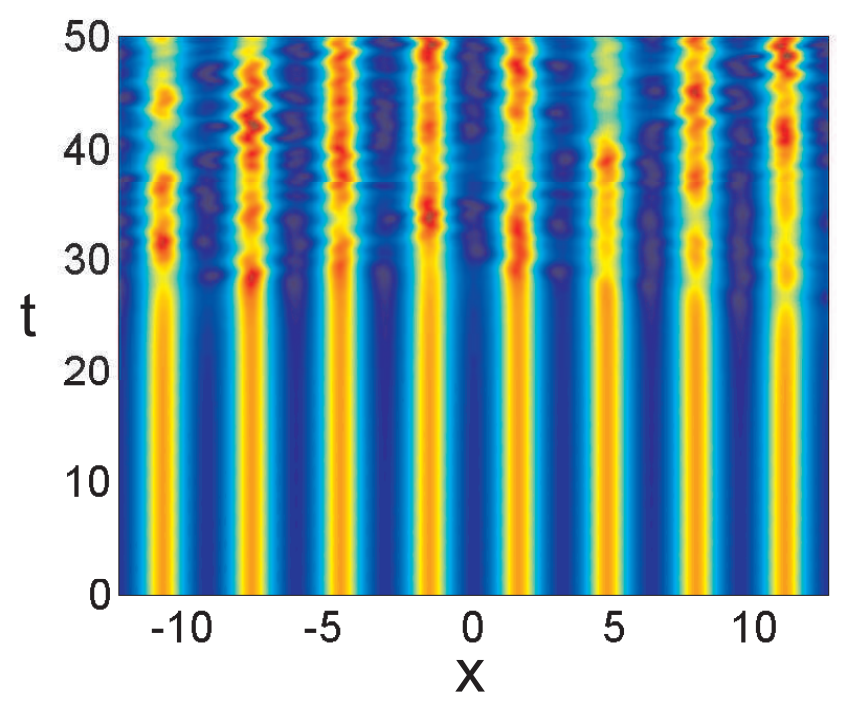

FIG. 4: (Color online) Temporal evolution of the BEC density for a current close to the critical value. Red color corresponds to the maximum density and blue color corresponds to the minimum density. The width of the window adjusted to show four periods of the initial field distribution. The parameters are $\mu=-2, v_{0}=\frac{8}{17}$, and $L=64 \pi$.

tion the maximum condensate density corresponding to the soliton peaks, located at $x=\pi / 2+\pi p$ where $p$ is an integer, is given by $\rho_{\max }=2 \mu / \min (U)$.

Since the phase difference $\theta$ is constant for adjacent solitons, the above expression of $J$ is similar to the supercurrent flowing at the zero voltage in a conventional superconductive Josephson junction (e.g., to the dc-Josephson effect) [27]. This suggests the interpretation of the fragmented condensates as an array of Josephson junctions and the persistent current as a BEC manifestation of the dc-Josephson effect [27]. The depleted areas between solitons (i.e., where the interaction is repulsive) play the role of insulating barriers of conventional Josephson junctions. Following this analogy we can say that for stable symmetric ground states the depleted areas are the analogous of 0 -junctions, while for the stable asymmetric states the depleted areas correspond to $\pi$ junctions. Also note that the role of the electric potential across the junction is played by the difference of chemical potentials between neighboring solitons. Since the fragmented state is a stationary state, it has a fixed chemical potential and, therefore, the flow of particles across the depleted regions (supercurrent) in the analogy also occurs in to the presence of zero voltage. Following this analogy, one can also expect that the nonzero-current stationary solutions become unstable and disappear when the current approaches a critical value (critical current). This is shown in Fig. 4 where the temporal evolution of the BEC density is depicted for a current very close to its critical calue. We see that in this case the condensate becomes very sensitive to perturbations and develops instabilities that eventually lead to its destruction (see the next sec- 
tion). We also observe that the critical current decays exponentially with the height and width of the effective nonlinear barrier, although it never vanishes completely.

\section{SOUND WAVES OF PERIODIC SOLITON}

The time evolution of sound waves against nonlinear periodic backgrounds of the form (13) is governed by the linear equation

$$
\begin{array}{r}
i \varphi_{t}=-\varphi_{x x}-2 i v_{0} \varphi_{x}+\left(2 U(x)\left|f_{v_{0}}\right|^{2}+v_{0}^{2}-\mu\right) \varphi \\
+U(x) f_{v_{0}}^{2} \varphi^{*} .
\end{array}
$$

Looking for a monochromatic excitation, $\varphi=$ $A(x) e^{-i(\omega+i \gamma) t}+B^{*}(x) e^{i(\omega-i \gamma) t}$, where $\omega(k)$ and $\gamma(k)$ are real, and splitting exponents of the respective ODEs one obtains the system of coupled equations with periodic coefficients:

$$
\begin{array}{r}
(\omega+i \gamma) A=-A_{x x}-2 i v_{0} A_{x}+ \\
\left(2 U(x)\left|f_{v_{0}}\right|^{2}+v_{0}^{2}-\mu\right) A+U(x) f_{v_{0}}^{2} B \\
-(\omega+i \gamma) B=-B_{x x}-2 i v_{0} B_{x}+ \\
\left(2 U(x)\left|f_{v_{0}}\right|^{2}+v_{0}^{2}-\mu\right) B+U(x) f_{v_{0}}^{* 2} A .
\end{array}
$$

These equations allow (due to the Floquet theorem) for solutions of the form $\sim \varphi_{k}(x) e^{i k x}$ with $\varphi_{k}(x)$ a periodic solution of Eq. (15) [it is worth noting, that the period of the function $f_{v_{0}}$ is not necessarily equal to the period of the nonlinear potential $U(x)$ ] because the coefficients in Eqs.(15) are $2 U(x)\left|\psi_{0}\right|^{2}$ and $U(x) \psi_{0}^{2}$, with the background state $\psi_{0}$ a periodic function of $x$. [In this paper we restrict our consideration to periodic backgrounds only].

The eigenvalues and the corresponding eigenfunctions can be found numerically: $\omega(k)$ yields the phase and the group velocities of the sound waves, while $\gamma(k)$ defines the stability of the ground state, the instability corresponding to positive $\gamma(k)$ (note that since the problem is Hamiltonian the real part $\gamma$ can appear only in pairs $\pm \gamma)$.

Now let us turn to the stability of the backgrounds with zero current whose spectrum is symmetric with respect to the transformation $k \rightarrow-k$. First, we consider the symmetric modes corresponding to the curve 1 in Fig. [1(b). In this case the period of the solution coincides with the period of the nonlinear potential and the width of the Brillouin zone of the linearized problem is equal to 2 . The solutions belonging to the lower branch of the bifurcation diagram are stable. The typical spectrum of the linear excitation is shown in Fig. [5(a). The spectrum is pure real and is of Bogoliubov type so $v_{g}=\partial_{k} \omega \neq 0$ at $k=0$. At the folding point the background becomes unstable, a fact that is confirmed by our spectral analysis. In particular, we find that the instability is generated by the merging of the lowest branches of the dispersion curve [i.e., the ones corresponding to lower values of $|\omega(k)|)$ ], (a)

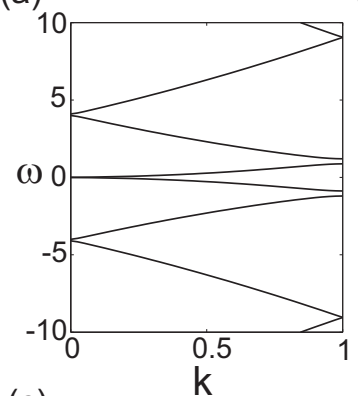

(c)

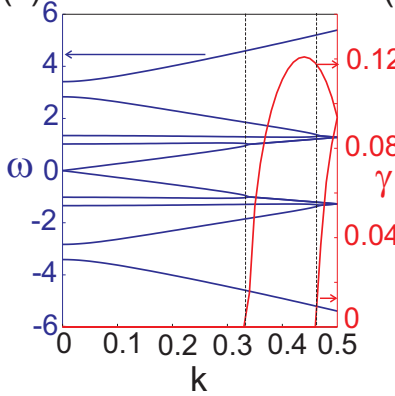

(b)

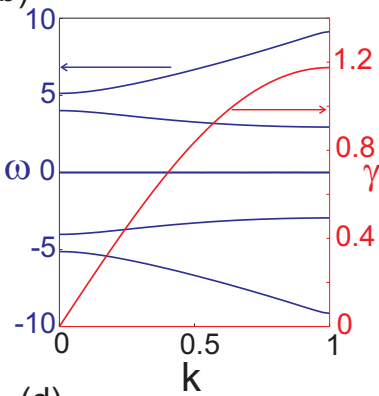

(d)

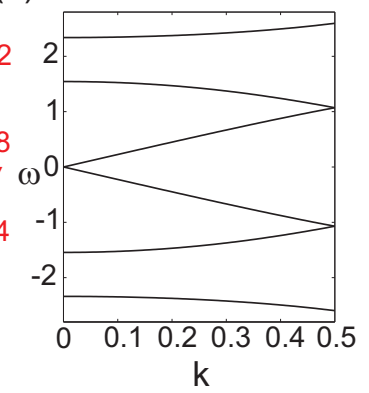

FIG. 5: (Color online) Sound wave spectra for the symmetric mode corresponding to curve 1 in Fig. 囵(b) at $\mu=0.04$ (a) and at $\mu=-2$ (b) on the lower and upper branches of the bifurcation curve, respectively. Panels (c) and (d) refer to sound wave spectra for backgrounds modes taken on curve 2 of Fig. 1(b) at chemical potentials $\mu=0$ and $\mu=-2$, correspondingly. The black and blue curves correspond to $\omega(k)$ (left vertical axis) and the red curves correspond to $\gamma(k)$ (the right vertical axis).

as illustrated in Fig. 5(b) for the case $\mu=-2$ for which branches are completely merged.

Next we consider the antisymmetric backgrounds corresponding to curve 2 in Fig. [1(b). In this case the coefficients in the linearized Eq.(15) have the period $\pi$ equal to the period of the potential only if the state is described by a pure real function $\Psi$, i.e., if the background has zero current. In the general case the period is equal to $2 \pi$ and the width of the Brillouin zone is equal to 1 . The spectra of the linear excitations on antisymmetric backgrounds are shown in Figs. 5 (c) and 5 (d).

The spectra shown in Fig. 5 reveal Bogoliubov structure; however, they behave differently as far as the stability concerns. Specifically, the states with relatively low particle density are unstable; see Fig 5. (c). It is seen that there are two pairs of merging modes, and the instability appears exactly at the wavevectors where two modes merge. On the other hand, at some threshold density $\rho \approx 3.4$ (the corresponding chemical potential $\mu \approx-0.2$ ) of the particles the antisymmetric state becomes stable; see the spectrum of the stable state shown in Fig. [5(d). The peculiarity of this state stems from the fact that the ground state is strongly depleted in the areas with repulsive nonlinear interatomic interaction. Therefore, for large negative chemical potentials some excitations can be considered as motion of weakly interacting 
drops of the condensate and this interaction becomes exponentially weak for large negative $\mu$. It means that the eigenvalues appear in the spectrum with very small absolute values and the chain of the droplets becomes very flat so even relatively weak external action results in the significant motion of the mutual phases of the droplets. Therefore, in this case even relatively weak external perturbation can result in the strongly nonlinear dynamics and in the decay of the condensate. As a result, even relatively weak perturbations can destroy stability due to nonlinear effects, while the state remains linearly stable.

We now consider how the stability of the antisymmetric state changes when $v_{0}$ deviates from zero and the ground state has nonzero current. In general, the period of the coefficients is equal to $2 \pi$; therefore, the width of the zone is equal to 1 . However, as mentioned above, at $v_{0}=0$ the period of the coefficients in the linearized equation is actually equal to $\pi$; - see Fig[6(a). For nonzero $v_{0}$ the period becomes equal to $2 \pi$ and gaps appear in the spectrum, as one can see from Figs. 6(b) and 6(c). One can also see that the symmetry $k \rightarrow-k$ becomes broken for nonzero current. For relatively small values of $v_{0}$ the background remains stable and thus one can claim existence of stable ground states with nonzero current.

At some intermediate value of $v_{0}$ the modes start to merge, generating instability. The onset of the instability goes to the Josephson critical current with the depletion, but for finite depletion the instability appears at the current lower the critical one. The development of the instability is illustrated in Fig. 4 . We took the initial condition in the form of the numerically found stationary solution perturbed by weak noise and performed the modeling. One can see that the noise destroys the state during relatively short time. At $v_{0}=1$ the states transform into the symmetric mode considered above. As noted, this state is unstable. The gaps at the boundary of the zone become closed because the period of the coefficients becomes equal to $\pi$.

Finally, we remark that the fact that the obtained spectra are of Bogoliubov type means that a perturbation moving (with subcritical velocity) with respect to the condensate does produce scattering (a certain amount of scattering may be seen, however, shortly after the introduction of the perturbation). This applies to the case of a perturbation that is resting with respect to the nonlinear potential, a problem to be addressed in the next section. The more general case of perturbations moving with respect to both the nonlinear lattice and the condensate will be considered elsewhere.

\section{SUPERFLUIDITY OF BOSE-EINSTEIN CONDENSATE}

It is well known that the scattering of the particles can be interpreted as Cherenkov synchronism between an obstacle and the propagating eigenmodes of the electron
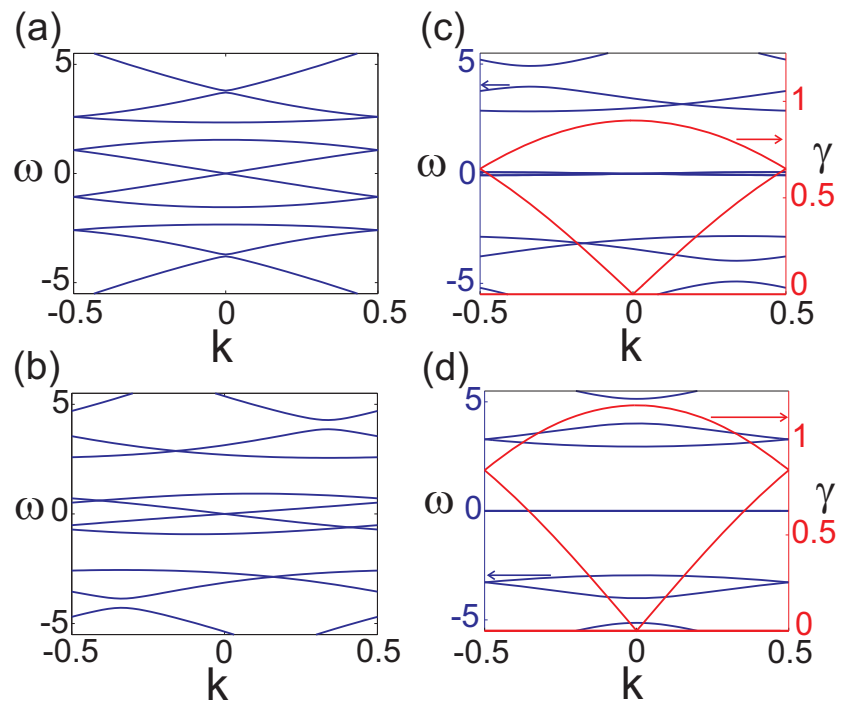

(d)

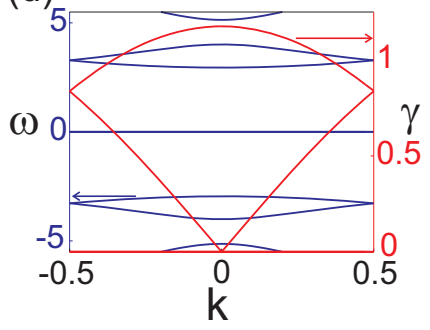

FIG. 6: (Color online) Sound wave spectra for the state with $\mu=-2$ and the quasivelocities $v_{0}=0$ (a), $v_{0}=0.33(\mathrm{~b})$, $v_{0}=0.66(\mathrm{c})$ and $v_{0}=1(\mathrm{~d})$. The antisymmetric state (a) and the symmetric one (d) modes correspond curves 2 and 1 in Fig. 11 respectively. Blue curves correspond to imaginary part $\omega$ of the eigenvalue (left vertical axis) and the red curves to the real part $\gamma$ of the eigenvalues (right vertical axis).

gas. When the system is described by the Schrödinger equation, then a localized obstacle can be represented as localized linear potential in the equation. For the linear Schrödinger equation, with the dispersion of the elementary excitations parabolic, the synchronism with a moving obstacle is inevitable unless the obstacle is resting with respect to the condensate. However, in the case of nonlinear Schrödinger equation with an uniform repulsive (defocusing) nonlinearity the dispersion becomes linear at low $k$, implying the existence of a critical velocity. The scattering occurs only if the velocity of the obstacle is larger than the critical velocity.

In the previous section we have shown that there exist the spectra of linear excitations in Bose-Einstein condensates with spatially inhomogeneous nonlinear interaction of Bogoliubov kind with nonzero $v_{g}$ at $k=0$. Therefore, one can expect that at least an obstacle resting relatively to the nonlinear periodical potential will not lead the scattering of the Bose-Einstein condensate flowing in the system (the condensate is flowing relatively to both the periodical potential and the obstacle). To confirm this hypothesis we performed numerical simulations of the condensate flowing in the large annular system with periodic nonlinear potential. The results are summarized Fig. 7 ,

One can see that for the stationary state the current is evenly distributed in space. If an obstacle leads to scattering, then the distribution of the current becomes inhomogeneous and, after some time, the averaged current tends to zero. We always calculated the distributions of currents assuming the initial condition the antisym- 
metric ground state discussed above and perturbing the equation by localized linear potential resting in respect to the periodical nonlinear potential. We carried out the simulations for very long time and no continuous scattering has been observed; the dependence of the current averaged over space is shown in Fig. 7(c).

The results of the numerical simulations indicate that introduction of the obstacle leads to very small perturbation of the current and the perturbation does not grow in time. This phenomenon is not sensitive to the relative position of the obstacle in respect to the nonlinear periodical potential; see Fig. 7 . We also verified that the perturbation of the current does not grow at arbitrary point of the system. For comparison, we show in the bottom part of Fig. Z(c) the dependence of the averaged current in the system with the same average particle density and the same initial current but without nonlinear interatom interaction. It is evident that the current oscillates and that the calculation time is considerably longer than the characteristic scattering time. The oscillation of the current in the linear system is, of course, periodic. Therefore, we can conclude that superfluidity of the BEC is observed in the system with periodic nonlinear potential.

\section{CONCLUSION}

We have explored the combined effect of nonlinearity and periodicity of nonlinear inter-atomic interaction in the toroidal traps where the scattering length is periodically modulated along the ring. First, we systematically studied periodic matter waves for both stationary states and states carrying nonzero current. The solutions found numerically have been parametrized by a chemical potential. We have shown for stationary states that when the nonlinear interaction changes its sign the condensate exists in the form of interacting drops localized in the areas with attractive interaction which rather counterintuitively implies the existence of the condensate with negative chemical potential despite of the dominating potential being repulsive. We also studied numerically the states with non-zero current and their relation to the stationary states. We have shown that the current depends periodically on the phase gradient and displays a strong fragmentation for large negative values of the chemical potential when the condensate is significantly depleted in the areas with repulsive interatomic interaction. We interpreted the fragmented condensates as Josephson $\pi$ junctions and the presence of the persistent current as a BEC analog of dc-Josephson effect which in contrast to a standard Josephson junction occurs in the system with and attractive effective inter-atomic potential.

Simultaneously, we studied sound waves propagating against the background. By inspecting the spectra of linear excitations on the ground states we investigated the stability of the condensate and we have shown that the spectrum are of Bogoliubov type. We found that the (a)

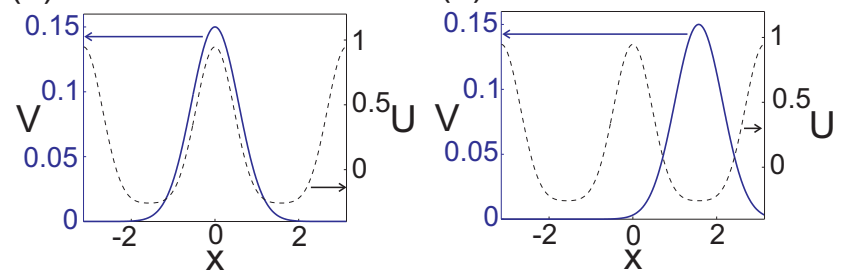

(c)
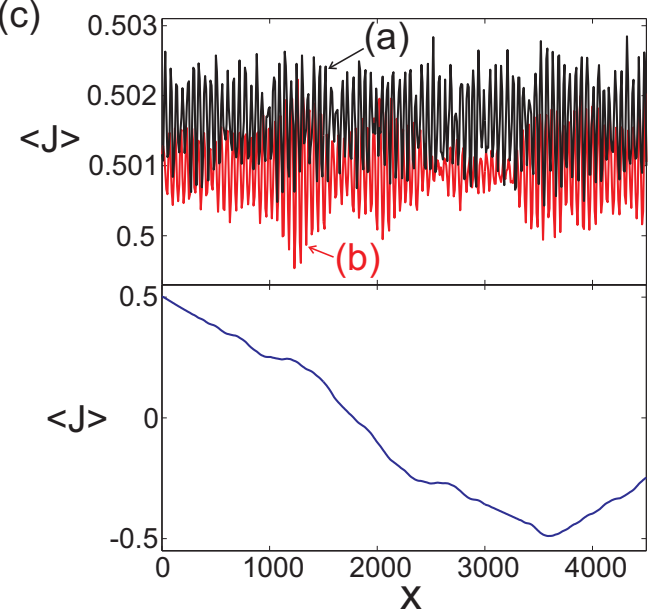

FIG. 7: (Color online) The dynamics of BEC in the presence of an obstacle described by the potential $V=$ $a_{0} \exp \left(-\frac{\left(x-x_{0}\right)^{2}}{w_{0}^{2}}\right)$. Panels (a) and (b) show the relative positions of the nonlinear periodical potential and the linear potential modeling an obstacle, $x_{0}=0$ for panel (a) and $x_{0}=\pi / 2$ for panel (b). The dynamics of the averaged current $\langle J\rangle$ is shown in the upper part of panel (c) for the relative position of the potentials shown in panels (a) (black line) and (b) (red line). The dynamics of the averaged current for the case without nonlinear interatom interaction is shown in the bottom part of panel (c). The averaged density and the initial velocity of the particles are the same as for the case with nonlinear interactions. Parameters used are $\mu=-2, v_{0}=\frac{1}{8}$, $a=0.15, w_{0}=0.8$, and $L=64 \pi$. Within the time scale shown in the figure the condensate makes about three rounds in the trap with a duration of each round of $512 \pi$ in dimensionless time units.

ground states consisting of interacting drops can be stable, which provides the strong evidence that these states and dc-Josephson effect can be experimentally observed. On the basis of numerical simulations we have demonstrated that superfluidity of Bose-Einstein condensate may take place in the system with inhomogeneous nonlinear interatom interaction. In particular, we have shown that a localized defect does not lead to the scattering of the condensate when the defect is at rest with respect to the nonlinear potential while the condensate is moving in respect to both the periodic potential and the defect. We have observed this behavior in the systems with both an effective repulsive and attractive interatom interaction. The case when the defect is moving in respect to both the lattice and the condensate will be considered in 
a separate paper.

\section{Acknowledgements}

AVY and VVK were supported by the FCT (Portugal) under the grant PEst-OE/FIS/UI0618/2011. AVY and VK were supported by the bilateral project. VK was partially supported by the Ministery of Education, Youth and Sports of the Czech Republic under the grant OC 09060. VVK was partially supported by the grant PIIFGA-2009-236099 (NOMATOS) within the 7th European Community Framework Programme. M. S. acknowledges support from Ministero dell' Istruzione, dell' Universitá e della Ricerca (MIUR) through the initiative "Programmi di Ricerca Scientifica di Rilevante Interesse Nazionale" (PRIN)-2008.
[1] A.J. Leggett, Quantum Liquids, Oxford Univ.Press, N.Y. (2006).

[2] M. F. Andersen, P. Clade, V. Natarajan, A. Vaziri, K. Helmerson, and W. D. Phillips, Phys. Rev. Lett. 97, 170406 (2006).

[3] C. Ryu, M. F. Andersen, P. Clade, V. Natarajan, K. Helmerson, and W. D. Phillips, Phys. Rev. Lett. 99, 260401 (2007).

[4] L. P. Pitaevskii, "Bose-Einstein condensation: the odd nonlinearity quantum mechanics", in "Nonlinear Waves: Classical and Quantum Aspects", Eds. A. Kh. Abdullaev and V. V. Konotop (Kluwer Academic Publishers, 2004) pp. $175-192$.

[5] H. Saito and M. Ueda, Phys. Rev. Lett. 93, 220402 (2004).

[6] Yu. V. Bludov and V. V. Konotop, Phys. Rev. A 75, 053614 (2007).

[7] J. Smyrnakis, M. Magiropoulos, G.M. Kavoulakis, and A.D. Jackson, Phys. Rev. A 82, 023604 (2010).

[8] B.B. Baizakov, B. Malomed, M. Salerno, Phys. Rev. E 74, 066615 (2006).

[9] B.P. Anderson and M.A. Kasevich, Science 282, 1686 (1998).

[10] V. Schweikhard, S. Tung, and E. A. Cornell, Phys. Rev. Lett. 99, 030401 (2007).

[11] R. A. Williams, S. Al-Assam, and C. J. Foot, Phys. Rev. Lett. 104, 050404 (2010).

[12] P. O. Fedichev, Y. Kagan, G. V. Shlyapnikov, and J. T. M. Walraven, Phys. Rev. Lett., 77, 2913 (1996).

[13] M. Salerno, V.V. Konotop, and Yu.V. Bludov, Phys. Rev. Lett. 101, 030405 (2008).

[14] Yu.V. Bludov, V. V. Konotop, and M. Salerno, Phys. Rev. A 80, 023623 (2009).

[15] Yu.V. Bludov, V. V. Konotop, and M. Salerno, Europhys. Lett. 87, 20004 (2009); J. Phys. B: At. Mol. Opt. Phys. 42105302 (2009).
[16] H. Sakaguchi and B. A. Malomed, Phys. Rev. E 72, 046610 (2005); Phys.Rev. E 73, 026601 (2006).

[17] F. Kh. Abdullaev and J. Garnier, Phys. Rev. A 72, 061605 (2005).

[18] H. L. F. da Luz, F. Kh. Abdullaev, A. Gammal, M.Salerno, and L. Tomio, Phys. Rev. A 82, 043618 (2010).

[19] N. G. Berloff and V. M. Perez-Garcia, arXiv:1006.4426v2.

[20] Y. V. Kartashov, B. A. Malomed, and L. Torner, Rev. Mod. Phys. 83, 247 (2011)

[21] S. Gupta et al., Phys. Rev. Lett. 95, 143201 (2005); A. S.Arnold, C. S. Garvie, and E. Riis, Phys. Rev. A 73, 041606(R) (2006).

[22] R. Yamazaki, S. Taie, S. Sugawa, and Y. Takahashi, Phys. Rev. Lett. 105050405 (2010).

[23] K. Henderson, C. Ryu, C. MacCormick1 and M.G. Boshier, New J. Phys. 11, 043030 (2009).

[24] D.S. Petrov and G.V. Schlyapnikov, Phys. Rev. A 64, 012706 (2001).

[25] E. M. Wright, J. Arlt, and K. Dholakia, Phys. Rev. A 63, 013608 (2001); B. P. Anderson, K. Dholakia, and E. M. Wright, Phys. Rev. A 67, 033601 (2003).

[26] Chao Hang and V. V. Konotop, Phys. Rev. A 83, 053845 (2011)

[27] A. Barone, G. Paterno, Physics and Applications of the Josephson Effect, John Wiley, N.Y. (1982).

[28] V. A. Brazhnyi and V. V. Konotop, Mod. Phys. Lett. B 18, 627 (2004).

[29] Yu. V. Bludov and V. V. Konotop, Phys. Rev. A 74, 043616 (2006).

[30] F. Bloch, Phys. Rev. A 7, 2187 (1976).

[31] Solution corresponding to quasi-periodical or nonperiodical chains of solitons are also possible but they are out of the scope of this paper 